\title{
Re-emergence of chronic obstructive pulmonary disease: it is time to think COPDifferently
}

Augustine Tee, MBBS, FRCP

ABSTRACT Knowledge of chronic obstructive pulmonary disease (COPD) as a common, preventable and treatable condition has advanced in the last two decades, as evidenced by the increase in scientific literature. Tobacco smoking still remains a predominant risk factor for COPD. Thus smoking cessation management should be obligatory in every case. Although spirometry is integral to the diagnosis of COPD, one should also be aware of its limitations. COPD is a chronic disease associated with comorbidities that define its extrapulmonary manifestations. Systemic inflammation provides the biological link, while exacerbations play a prominent role in the current approach to disease evaluation. This paper reviews the latest Global Initiative for Chronic Obstructive Pulmonary Disease revision, focusing on the paradigm shift in assessment that would directly influence therapeutic decisions. Also discussed are the newer drugs and combinations of existing inhaler therapies that now present clinicians with more options, as well as bronchoscopic interventions that may perhaps offer a lower-morbidity alternative than surgical lung volume reduction. Finally, this review highlights how integrated care models can bridge the gap between components and complete a comprehensive sphere of COPD care.

Keywords: chronic airflow obstruction, chronic bronchitis, chronic obstructive pulmonary disease, COPD pulmonary emphysema

\section{INTRODUCTION}

Chronic obstructive pulmonary disease (COPD) is starting to receive the attention that has eluded it for the last century, with more scientific research conducted and published in the last 20 years than in all of its known history combined. This came about partly from the concerted efforts of the Global Initiative for Chronic Obstructive Lung Diseases (GOLD), a collaborative effort among the United States' National Heart, Lung, and Blood Institute, National Institutes of Health and World Health Organization. Since 1997, GOLD committees have worked with healthcare professionals and public health officials around the world to raise awareness of COPD, as well as to improve its prevention and treatment. ${ }^{(1)}$

The affliction that we now recognise as COPD was thought to be first described in 1837 by Laënnec, the inventor of the stethoscope. Noting that the postmortem lungs of sufferers were hyperinflated, Laënnec deduced that "the chief reason of this affection having been so completely overlooked is that it is in some sort merely the exaggeration of the natural condition of the viscus." In an era where cigarette smoking was rare, the finding correlated with asthma, which was considered a 'nervous' condition then. ${ }^{(2)}$ It was not until 1966 that a new name for an old malady, encompassing chronic bronchitis, asthmatic bronchitis and emphysema, came to be included under the rubric 'chronic obstructive pulmonary disease', which became widely adopted. ${ }^{(3)}$

Fast-forwarding to this millennium, COPD is still a largely underappreciated medical condition. At least half of all patients with COPD in the United States are undiagnosed.(4) Even when diagnosed, undertreatment is prevalent, with $30 \%-70 \%$ of diagnosed individuals receiving either no treatment or suboptimal treatment. ${ }^{(5,6)}$ In spite of the potential under-detection, as of 2011, COPD was the 7th and 8th most common cause of hospitalisation and mortality, respectively, in Singapore. ${ }^{(7)}$

\section{SMOKER'S DISEASE OR DISEASED SMOKER?}

With smoking proven to be strongly associated with a decline in lung function, as reported in the classic paper by Fletcher and Peto, ${ }^{(8)}$ the aetiology of COPD was mainly attributed to tobacco exposure until recently. Evidence of nonsmoking COPD occurs with a wide variation in prevalence and is reported in at least one out of four diagnosed patients, with a much higher degree of disease burden than previously believed. ${ }^{(9)}$ Risk factors for COPD in nonsmokers may include genetic factors, longstanding asthma, outdoor air pollution (from traffic and other sources), environmental tobacco smoke exposure, biomass smoke exposure, occupational smoke exposure, diet, recurrent respiratory infection in early childhood and tuberculosis. ${ }^{(10)}$ Nonsmoking COPD is a neglected entity typically excluded in clinical trials, with a lack of characterisation of its profile. Such a misrepresentation occurs despite COPD being defined as a disease of exposure to noxious stimuli, and not cigarette smoke in particular. More studies to determine the role and contribution of these other aetiological factors toward the COPD state are much anticipated.

Tobacco smoking has been linked to COPD since the 1950s and is widely known as the single most important risk factor for COPD. When implicated as an obvious cause, it is frequently difficult to eradicate in the patient. Cigarette smoking, or more accurately, nicotine dependence is an addiction. 
It constitutes a second disease that not only occurs together with COPD, but requires a different management approach with different treatment options - some of which may not be options that physicians are comfortable with. ${ }^{(11)}$ It is reassuring that smoking cessation continues to be a healthcare priority locally, with Singapore hosting the 15th World Conference on Tobacco or Health in March 2012 and the Health Promotion Board-Ministry of Health releasing the Clinical Practice Guidelines on Treating Tobacco Use and Dependence in 2013. .12) $^{(2)}$ All healthcare practitioners have an inherent responsibility toward their patients who smoke. In order to help patients succeed in overcoming nicotine addiction, we need to debunk the myths and superstitions surrounding smoking cessation and assist patients' efforts through behavioural management and pharmacotherapy.

\section{OBSTRUCTIVE BUT NOT FULLY REVERSIBLE?}

In the most recent revision of the GOLD guidelines in 2011, spirometry was made a requirement to confirm the clinical diagnosis of COPD, further emphasising the importance of this test modality. The use of spirometry prevents misdiagnosis and ensures proper evaluation of the severity of airflow limitation. ${ }^{(13)}$ However, access to reliable spirometry continues to be lacking in many countries, resulting in the underdiagnosis of COPD in these countries. In a 2012 worldwide survey on early COPD diagnosis, the most frequently cited priority was the need to improve the use of spirometry in primary care. ${ }^{(14)}$ Diagnosing COPD without a spirometer reading may be comparable to an attempt to detect hypertension without a sphygmomanometer blood pressure reading.

Furthermore, the physician faces other challenges in the spirometric assessment of COPD. Since COPD is defined as a condition of airflow obstruction, a spirometric obstructive pattern of forced expiratory volume in the first second $\left(\mathrm{FEV}_{1}\right)$ /forced vital capacity (FVC) of less than 0.7 determines this abnormality. As the pathological changes of COPD may appear years before physiological airflow obstruction manifests, a normal spirometry reading or $\mathrm{FEV}_{1}$ does not completely rule out COPD or other lung conditions. In fact, symptomatic individuals with smoking exposure but normal airflow have been found to be at risk of COPD, with some reported to show evidence of emphysema on computed tomography (CT). ${ }^{(15)}$ In the COPDGene population, $10 \%$ of patients have suggestion of a restrictive airflow pattern with a normal $\mathrm{FEV}_{1} / \mathrm{FVC}$ ratio and a low $\mathrm{FEV}_{1}{ }^{\left({ }^{16)}\right.}$ This heterogeneous group had significant symptoms and functional limitation, and is also likely to have a variety of underlying aetiologies beyond increased body mass index (BMI). ${ }^{(16)}$ There is now greater awareness among experts in the field regarding the need to look beyond the traditional spirometric definitions of COPD. Nevertheless, as $\mathrm{FEV}_{1}$ is well studied, standardised, repeatable and has prognostic bearing, it still remains the measure of primary lung function outcome in most asthma and COPD studies, and is used as the basis of COPD drug registration by most health authorities.

The traditional teaching that bronchodilators do not significantly reverse airflow obstruction in COPD is no longer thought to reflect the true nature of diseased airways. Many COPD patients fulfill these spirometric criteria for bronchodilator response, whereas some asthmatics do not (i.e. irreversible asthma). This has led to a change in the description of COPD to that of an airway disease that is not fully reversible. In fact, GOLD has removed the reversibility description from its definition of COPD. In essence, the diagnosis of airflow obstruction using a decreased $\mathrm{FEV}_{1} / \mathrm{FVC}$ ratio of less than 0.70 is more essential, with only post-bronchodilator $\mathrm{FEV}_{1}$ needed to classify severity of airflow limitation, without the need for gauging the presence or absence of a bronchodilator response. With this imperfection in mind, some experts have suggested the use of CT examinations ${ }^{(14)}$ to provide a useful measure of the presence and extent of emphysema, airway disease, and more recently, pulmonary vascular disease for clinical correlation. CT, however, will not detect chronic bronchitic COPD in a patient without emphysema, and thus its overall utility requires further study. In smokers, emphysema distribution on CT has been shown to be associated with BMI, $\mathrm{FEV}_{1}$, severity of dyspnoea and prognostic index (i.e. BODE [BMI, airflow obstruction, dyspnoea and exercise capacity] index).(17)

\section{PULMONARY AND EXTRAPULMONARY - TRULY SYSTEMIC}

Comorbidities are now well accepted in the assessment and management of COPD, even though they are often underdiagnosed and undertreated. Many identified comorbid conditions are present at a higher frequency in COPD cohorts than in the general adult population, although common risk factors such as ageing, smoking and obesity are contributing factors. The comorbid conditions that may be less noticeable are cardiovascular disease, malignancy, metabolic syndrome, osteoporosis, depression and other systemic diseases. They affect the prognosis ${ }^{(18)}$ of the individual patient with COPD, frequently mimicking and confusing symptom presentation, and may even be the dominant problem. There is early evidence that a multidimensional COPD management approach that takes comorbidities into account may improve treatment response and reduce mortality in patients with COPD. ${ }^{(19)}$ Therefore, cardiovascular, metabolic, and other relevant examinations need to be considered when assessing patients with COPD. Lung function testing may have a role in the assessment of cardiovascular and other diseases. As evidence of the close association between the comorbidities and COPD, some non-respiratory drug therapies such as statins and beta-blockers have been shown to positively impact morbidity and mortality in patients with COPD. Rigorous clinical trials should be awaited before making recommendations on comorbidity management in the context of COPD. Meanwhile, 
these conditions are treated based on their specific disease guidelines.

A central theme in the pathogenesis of these comorbid conditions in COPD is systemic inflammation. Chronic inflammation can be demonstrated in small airways and lung parenchyma via tissue biopsies and sputum analyses. This type of inflammation persists despite the removal of the noxious irritant and has a characteristic different from that found in asthma, with the former being more steroid-resistant. Therapeutic agents such as inhaled corticosteroids, which are highly effective and essential in most patients with asthma, may not be optimal in patients with COPD who show limited efficacy and modest reductions in exacerbation rates. Furthermore, the inflammatory effects extend beyond the lungs. In particular, the presence of peripheral blood inflammatory markers, cardiovascular mortality, muscle dysfunction and wasting suggests that this supposed 'spill-over' effect of inflammation may play a wider role. ${ }^{(20,21)}$

\section{AN EXACERBATION IS NOT AN \\ EXAGGERATION}

Exacerbation is a key milestone in the natural history of a patient with COPD. Lung and systemic inflammation intensifies during most exacerbations, which are mainly triggered by bacterial or viral infections, or environmental pollutants. Interestingly, the cause of an exacerbation can be varied, overlapping and occasionally unknown. (22) An exacerbation is generally an acute deterioration of COPD symptoms. GOLD defines an exacerbation as a worsening of symptoms from its day-to-day variation, requiring a change in medication. This often leads to medical consults or hospitalisations, which constitute a healthcare economic burden. To the patient, an exacerbation leads to an accelerated decline in lung function and reduced physical activity; it negatively impacts one's health-related quality of life, resulting in significant morbidity and mortality. ${ }^{(23)}$ Thus, recognition of an exacerbation, which enables early effective treatment and reduction of future risk of exacerbation, is integral to the present day COPD approach. We should no longer accept episodic care of COPD exacerbations as the norm.

A COPD patient's history of exacerbations has been shown to identify the particular patient phenotype at risk for future episodes of exacerbations. In the multicentre ECLIPSE (Evaluation of COPD Longitudinally to Identify Predictive Surrogate Endpoints) study in 2010,(24) the frequency and association of exacerbation in 2,138 patients were evaluated over a period of three years. Not only did the exacerbations become more frequent and more severe with more advanced COPD, but the single best predictor of exacerbations across all GOLD stages was a history of exacerbations. This 'frequentexacerbation' phenotype appeared to be relatively stable over the three-year period and could be predicted on the basis of the patients' recall of previous treated events. This and other studies drove the 2011 GOLD recommendation to use

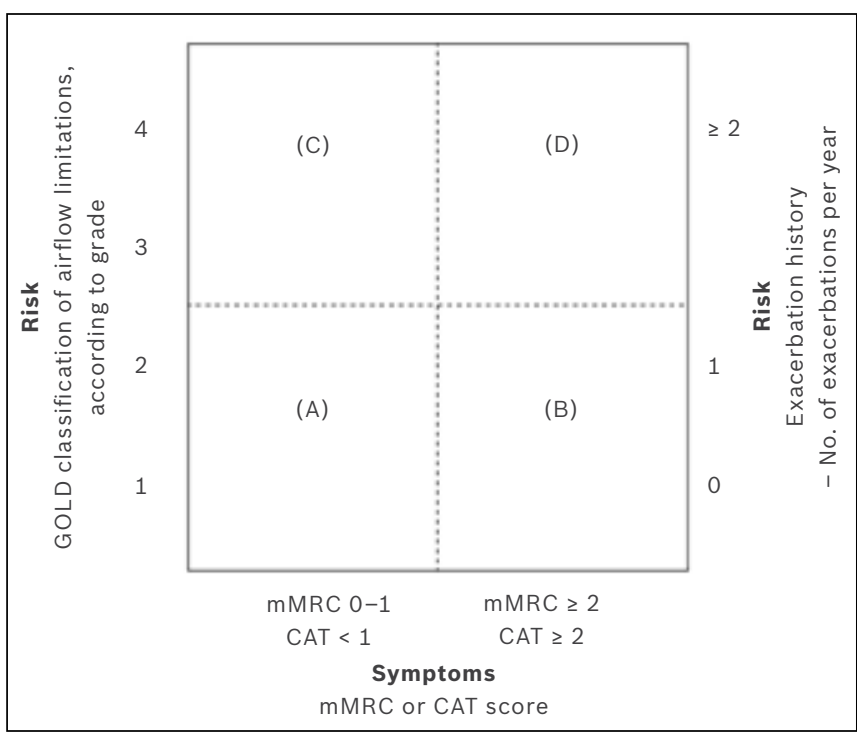

Fig. 1 Diagram shows the $2 \times 2$ matrix of the GOLD combined assessment of COPD [adapted from GOLD 2011 revision ${ }^{(11)}$ ]. CAT: COPD Assessment Test; GOLD: Global Initiative for Chronic Obstructive Lung Disease; mMRC: modified British Medical Research Council dyspnoea scale

history of exacerbations to guide future risk assessment in COPD. ${ }^{(23,25)}$

\section{A PARADIGM SHIFT IN GOLD}

This review is incomplete without comment on the 2011 GOLD revision $^{(13)}$ and the recent 2013 update. $^{(26)}$ A paradigm shift in diagnostic approach, clinical evaluation and therapeutic treatment of the disease as compared to its 2006 version, was boldly introduced in late 2011. The most significant changes in the document were in the evaluation of COPD, which now pivots around the impact of the symptoms, the future risk of exacerbations, the severity of airflow obstruction and the identification of comorbidities.

For the first time, the evaluation of breathlessness using the modified British Medical Research Council dyspnoea scale or the evaluation of quality of life using the COPD Assessment Test score $^{(27)}$ has a direct impact on therapeutic decisions. This, together with the evaluation of airflow obstruction grade with $\mathrm{FEV}_{1} \%$ predicted and the number of exacerbations in the past one year, results in a $2 \times 2$ matrix that groups patients into four groups (A, B, C and D) (Fig. 1), with separate drug treatments recommended for each group. In the past, the treatment recommendation of chronic stable disease followed a stepladder schedule that was correlated with airflow severity. The concept of COPD 'systemic effects' in earlier GOLD definitions has been replaced by that of 'comorbidities'. Additionally, it has been highlighted that cardiovascular diseases, diabetes mellitus, anxiety and depression, infections, lung cancer, metabolic syndrome and osteoporosis should be approached in a therapeutic manner, similar to any other clinical situation, regardless of the presence of COPD.

The 2011 GOLD revision is an evolution of recommendations based on cumulated scientific evidence. In particular, disease evaluation has changed from unidimensional airflow assessment 
to that of a multidimensional (symptoms, quality of life, exacerbations and airflow) matrix. This does not necessitate sophisticated technology, and can be applied in any clinical situation and place. Treatments can be instituted with confidence in order to gain early symptomatic relief and prevent future adverse events.

\section{INHALED DRUGS AND IMPLANTED VALVES}

The spectrum of drug armamentarium available for COPD treatment is set to expand in this decade. Established concepts of bronchodilatation and anti-inflammation have spurred drug development toward newer and more effective agents in each class. Short-acting beta-2-agonists as the sole medication should ideally belong only to the low-risk and least symptomatic patient with COPD. Long-acting beta-2-agonist (LABA) and ultraLABAs (e.g. indacaterol) have been shown to maintain superb bronchodilatation over extended periods of time, translating into a once-daily formulation. Long-acting antimuscarinic agents (LAMA) such as tiotropium are also a powerful once-daily therapy that provides excellent symptom relief and $\mathrm{FEV}_{1}$ improvement while reducing exacerbation rate and related hospitalisations, as shown in the four-year UPLIFT (Understanding Potential Long-Term Impacts on Function with Tiotropium) trial. ${ }^{(28)}$ Many other LABAs (e.g. vilanterol, oladaterol) and LAMAs (e.g. glycopyrronium, aclidinium, umeclidinium), as well as combinations of LABA-LAMA are now in the final stages of development or pending drug registration to be approved for use in COPD. Inhaled corticosteroid (ICS) and LABA combinations are familiar medications in the management of asthma, with well-evidenced scientific merit and clinical benefits. However, after many years, the role of ICS in COPD is still debatable. Nonetheless, the landmark threeyear TORCH (TOwards a Revolution in COPD Health) study(29) has shown that although there was no significant reduction in death from all causes in ICS-LABA treated patients, there was a reduction in the annual rate of exacerbations, as well as improved health status and spirometric values. Of note, although there was no difference in the incidence of ocular or bone side effects, ICS-treated patients had a higher probability of contracting pneumonia. However, the clinical significance of ICS-related pneumonia is still disputed. ${ }^{(30-32)}$ Roflumilast, a new oral phosphodiesterase-4 inhibitor, is an antiinflammatory drug approved for use in moderate to severe COPD patients with symptoms of chronic bronchitis and frequent exacerbations. This subset of COPD patients benefitted most in terms of lung function, symptom improvement and lower exacerbation rates; ${ }^{(33,34)}$ however, treated patients showed small, but significant, weight loss and gastrointestinal effects.

In 2003, the National Emphysema Treatment Trial (NETT) ${ }^{(35)}$ proved that in a highly selected group of COPD patients (upper lobe emphysema with low exercise capacity), a survival advantage could be gained by lung volume reduction surgery.
This surgery, however, carried significant morbidity of prolonged postoperative air-leaks and hospitalisation, ${ }^{(36)}$ and the trial results could not be easily replicated in centres with less experience in the modality. This led to the exploration of bronchoscopic methods of lung volume reduction using valves, coils and thermal vapour. ${ }^{(37)}$ So far, one-way Zephyr ${ }^{\circledR}$ endobronchial valves with lobar placement guided by a catheter-based collateral ventilation assessment seems to hold some promise of being feasible and safe, with long-term sustained improvement in lung function, symptoms and exercise tolerance. ${ }^{(38-40)}$ We await further larger trials in the United States and Asia before this modality can be routinely recommended.

\section{AN INTEGRATED CARE APPROACH}

Integrated care in the widest sense, as defined by World Health Organization, is a concept of bringing together inputs, delivery, management and organisation of services related to diagnosis, treatment, care, rehabilitation and health promotion. It combines the best of conventional and complementary healthcare. It undoubtedly centres on multidisciplinary collaboration, coordination of multiple sites of care, and engagement at multiple stages in the patient's disease history, i.e. from prevention and primary care to palliative and advanced care. Being a prevalent chronic disease, COPD should not simply be managed using pharmacotherapy, but with a more comprehensive approach that would be beneficial to both the patient and society. In short, the concept of integrated care encompasses six key components: selfmanagement support, clinical information systems, delivery system redesign, decision support (guidelines), healthcare organisation, and community resources. ${ }^{(41)}$ It is still unknown which of these six components are necessary for ideal management of the COPD patient. A systematic review suggests that two or more components are needed for optimal care. $^{(42)}$ Evidence-based literature pertaining to integrated care for COPD is still in early development as compared to well-known research findings on chronic disease management. With many different models of care in the United Kingdom, United States, Canada and Europe, it is clear that one size will not fit all. Examples of innovative methods of integrating COPD patient care include tele-healthcare, case managers, specialised non-hospital-based centres and interactive information technology platforms, among others. Even in Singapore, this small island nation, there are different integrated care models of COPD arising from the separate regional health clusters.

\section{CONCLUSION}

COPD is now more prevalent than before. There is a greater appreciation of the challenges of smoking cessation and nicotine dependence. Worldwide, the non-smoking population of COPD is getting more attention. Although spirometry is required to confirm COPD, early disease cannot be detected 
using this physiological test. Comorbidities, especially cardiovascular disease, metabolic syndrome and depression, are some of the extrapulmonary manifestations that can impact the presentation and prognosis of COPD. Exacerbations are an important part of COPD history, and they are included in the revised GOLD evaluation matrix. Airflow obstruction, symptoms and quality of life scores are the other contributors to this assessment. Hence, therapeutic objectives are guided by the assessment of the impact of COPD on the patient's symptoms and state of health, as well as the risk of future events (exacerbations and mortality). This places COPD treatment within the context of a more personalised clinical practice that is closer to the individual needs of each patient. With an integrated care concept assimilating best practices and innovating models of patient support, patients with COPD should no longer accept a nihilistic attitude from caregivers.

\section{REFERENCES}

1. Global Initiative for Chronic Obstructive Lung Disease. GOLD - the Global Initiative for Chronic Obstructive Lung Disease. Available at: www. goldcopd.org. Accessed November 29, 2013.

2. Petty TL. The history of COPD. Int J Chron Obstruct Pulmon Dis 2006; 1:3-14.

3. Fishman AP. One hundred years of chronic obstructive pulmonary disease. Am J Respir Crit Care Med 2005; 171: 941-8.

4. Mannino DM, Homa DM, Akinbami LJ, Ford ES, Redd SC. Chronic obstructive pulmonary disease surveillance-United States, 1971-2000. MMWR Surveill Summ 2002; 51:1-16.

5. Takahashi $\mathrm{T}$, Ichinose $\mathrm{M}$, Inoue $\mathrm{H}$, et al. Underdiagnosis and undertreatment of COPD in primary care settings. Respirology 2003; 8:504-8.

6. Criner GJ, Cordova F, Sternberg AL, et al. The national emphysema treatment trial (NETT): Part I: Lessons learned about emphysema. Am J Respir Crit Care Med 2011; 184:763-70.

7. Ministry of Health Singapore, Statistics, Singapore Health Facts [online]. Available at: http://www.moh.gov.sg/content/moh_web/home/statistics/ Health_Facts_Singapore.html. Accessed July 26, 2013.

8. Fletcher $\mathrm{C}$, Peto R. The natural history of chronic airflow obstruction. $\mathrm{Br}$ Med J 1977; 1:1645-8.

9. Salvi SS, Barnes PJ. Chronic obstructive pulmonary disease in non-smokers. Lancet 2009; 374:733-43.

10. Zeng G, Sun B, Zhong N. Non-smoking-related chronic obstructive pulmonary disease: a neglected entity? Respirology 2012; 17:908-12.

11. Raupach T, Merker J, Hasenfuss G, Andreas S, Pipe A. Knowledge gaps about smoking cessation in hospitalized patients and their doctors. Eur J Cardiovasc Prev Rehabil 2011; 18:334-41.

12. HPB-MOH Clinical Practice Guidelines 1/2013 Treating Tobacco Use and Dependence [online]. Available at: http://www.hpb.gov.sg/HOPPortal/ publication-article/HPB049610. Accessed July 26, 2013.

13. Vestbo J, Hurd SS, Agustı AG, et al. Global strategy for the diagnosis, management, and prevention of chronic obstructive pulmonary disease. GOLD Executive Summary. Am J Respir Crit Care Med 2013; 187:347-65.

14. International COPD coalition. Worldwide Survey on Early COPD Diagnosis [online]. Available at: http://www.internationalcopd.org/WCDMaterials/ Worldwide_Survey_on_Early_COPD_Diagnos is.aspx. Accessed July 26, 2013.

15. Rennard S, Thomashow B, Crapo J, et al. Introducing the COPD Foundation Guide for Diagnosis and Management of COPD, recommendations of the COPD Foundation. COPD 2013; 10:378-89.

16. Wan ES, Hokanson JE, Murphy JR, et al. Clinical and radiographic predictors of GOLDunclassified smokers in the COPDgene study. Am J Respir Crit Care Med 2011; 184:57-63.

17. Mair G, Miller JJ, McAllister D, et al. Computed tomographic emphysema distribution: relationship to clinical features in a cohort of smokers. Eur Respir J 2009; 33:536-42.
18. Divo M, Cote $\mathrm{C}$, de Torres JP, et al. Comorbidities and risk of mortality in patients with chronic obstructive pulmonary disease. Am J Respir Crit Care Med 2012; 186:155-61.

19. Luppi F, Franco F, Beghé B, Fabbri LM. Treatment of chronic obstructive pulmonary disease and its comorbidities. Proc Am Thorac Soc 2008; 5:848-56.

20. Balasubramanian VP, Varkey B. Chronic obstructive pulmonary disease: effects beyond the lungs. Curr Opin Pulm Med 2006; 12:106-12.

21. Sethi S, Mahler DA, Marcus P, et al. Inflammation in COPD: implications for management. Am J Med 2012; 125:1162-70.

22. MacDonald M, Beasley RW, Irving L, Bardin PG. A hypothesis to phenotype COPD exacerbations by aetiology. Respirology 2011; 16:264-8.

23. Celli BR, Barnes PJ. Exacerbations of chronic obstructive pulmonary disease. Eur Respir J 2007; 29:1224-38.

24. Hurst JR, Vestbo J, Anzueto A, et al. Susceptibility to exacerbation in chronic obstructive pulmonary disease. N Engl J Med 2010; 363:1128-38.

25. Groenewegen KH, Schols AM, Wouters EF. Mortality and mortality-related factors after hospitalization for acute exacerbation of COPD. Chest 2003; 124:459-67.

26. Vestbo J, Hurd SS, Agustí AG, et al. Global strategy for the diagnosis, management, and prevention of chronic obstructive pulmonary disease. GOLD executive summary. Am J Respir Crit Care Med 2013; 187:347-65.

27. Jones PW, Harding G, Berry P, et al. Development and first validation of the COPD Assessment Test. Eur Respir J 2009; 34:648-54.

28. Tashkin DP, Celli B, Senn S, et al. A 4-Year Trial of tiotropium in chronic obstructive pulmonary disease. N Engl J Med 2008; 359:1543-54.

29. Calverley PMA, Anderson JA, Celli B, et al. Salmeterol and fluticasone propionate and survival in chronic obstructive pulmonary disease. $\mathrm{N} \mathrm{Engl}$ J Med 2007; 356:775-89.

30. Malo de Molina RM, Mortensen EM, Restrepo MI, et al. Inhaled corticosteroid use is associated with lower mortality for subjects with COPD and hospitalised with pneumonia. Eur Respir J 2010; 36:751-7.

31. Singh S, Amin AV, Loke YK. Long-term use of inhaled corticosteroids and the risk of pneumonia in chronic obstructive pulmonary disease. A Metaanalysis. Arch Intern Med 2009; 169:219-29.

32. Janson C, Larsson K, Lisspers KH, et al. Pneumonia and pneumonia related mortality in patients with COPD treated with fixed combinations of inhaled corticosteroid and long acting $\beta 2$ agonist: observational matched cohort study (PATHOS). BMJ 2013; 346:f3306

33. Calverley PM, Rabe KF, Goehring UM, et al. Roflumilast in symptomatic chronic obstructive pulmonary disease: two randomised clinical trials. Lancet 2009; 374:685-94.

34. Fabbri LM, Calverley PM, Izquierdo-Alonso JL, et al. Roflumilast in moderate-to-severe chronic obstructive pulmonary disease treated with long-acting bronchodilators: two randomised clinical trials. Lancet 2009; 374:695-703.

35. Fishman A, Martinez F, Naunheim K et al; National Emphysema Treatmen Trial Research Group. A randomized trial comparing lung-volumereduction surgery with medical therapy for severe emphysema. N Engl Med 2003; 348:2059-73.

36. Criner GJ, Cordova F, Sternberg AL, Martinez FJ. The National Emphysema Treatment Trial (NETT) Part II: Lessons learned about lung volume reduction surgery. Am J Respir Crit Care Med 2011; 184:881-93.

37. Taneja A. Bronchoscopic interventions in the management of chronic obstructive pulmonary disease. Curr Opin Pulm Med 2013; 19:145-51.

38. Venuta F, Anile M, Diso D, et al. Long-term follow-up after bronchoscopic lung volume reduction in patients with emphysema. Eur Respir J 2012; 39:1084-9.

39. Herth FI, Noppen M, Valipour A, et al; International VENT Study Group. Efficacy predictors of lung volume reduction with Zephyr valves in a European cohort. Eur Respir J 2012; 39:1334-42.

40. Herth FJ, Eberhardt R, Gompelmann D, et al. Radiological and clinical outcomes of using ChartisTM to plan endobronchial valve treatment. Eur Respir J 2013; 41:302-8

41. Nici L, ZuWallack R, American Thoracic Society Subcommittee on Integrated Care of the COPD Patient. An Official American Thoracic Society workshop report: the Integrated Care of The COPD Patient. Proc Am Thorac Soc 2012; 9:9-18.

42. Adams SG, Smith PK, Allan PF, et al. Systematic review of the chronic care model in chronic obstructive pulmonary disease prevention and management. Arch Intern Med 2007; 167:551-61. 